Fiber Optic Sensors: Current Status and Future Possibilities

ISBN:978-3-319-42624-2

Smart Sensors, Measurement and Instrumentation, Volume 21, 2017, pp 241-267.

DOI: $10.1007 / 978-3-319-42625-9 \_12$

\title{
LONG PERIOD GRATING BASED FIBRE OPTIC CHEMICAL SENSORS
}

\author{
Sergiy Korposh ${ }^{1}$, Seung-Woo Lee ${ }^{2}$ and Stephen James ${ }^{3}$ \\ ${ }^{1}$ Advanced Optics Group, The University of Nottingham, UK; \\ ${ }^{2}$ The University of Kitakyushu, Japan; \\ ${ }^{3}$ Engineering Photonics, Cranfield University, UK.
}

\begin{abstract}
The principle of operation of optical fibre long period grating (LPG) sensors is described. In particular, the chapter explores the use of LPGs as a chemical sensing platform, discussing the fabrication of LPGs and the various approaches that have been employed to modify the cladding of the LPG and thus sensitise the LPG. Examples of the practical application of LPG chemical sensors are provided.
\end{abstract}

\section{INTRODUCTION}

The development of optical fibre long period grating (LPG) based sensors is an area of considerable research activity, with the interest driven by their sensitivity to a number of parameters, principally temperature, strain, bending and the surrounding refractive index, by the potential for multi-parameter sensing using as single optical element, and by the ability to tune the sensitivity of the devices by appropriate choice of period. Over the past decade there has been a particular interest in the deposition onto the cladding of optical fibres containing LPGs of chemically sensitive coatings that change their refractive index in response to specific chemical stimuli. This chapter will focus on the use of LPGs as a chemical and bio-sensing platform, outlining approaches used to fabricate LPGs, the techniques used to deposit materials onto the optical fibres, means for optimising sensitivity, and examples of their use. The chapter will draw upon work that has been conducted as collaboration between teams at the Universities of Kitakyushu, Cranfield and Nottingham.

An LPG, shown schematically in figure 1(a), is a periodic modulation of the properties of an optical fibre, which facilitates the coupling of light from the propagating core modes to a set of co-propagating cladding modes. The high attenuation of the cladding modes results in a series of resonance bands in the transmission spectrum of the optical fibre, as shown in figure 1(b). The resonance bands centred at wavelengths satisfying the phase matching condition:

$$
\lambda_{\mathrm{x}}=\left(\mathrm{n}_{\text {core }}-\mathrm{n}_{\text {clad }(\mathrm{x})}\right) \Lambda
$$

Where $\lambda_{x}$ represents the wavelength at which light is coupled to the $\mathrm{LP}_{0 \mathrm{x}}$ cladding mode, $n_{\text {core }}$ is the effective refractive index of the mode propagating in the core of the fibre, $n_{\text {clad }(x)}$ is the effective index of the $\mathrm{LP}_{0 \mathrm{x}}$ cladding mode and $\Lambda$ is the period of the $\mathrm{LPG}$. 
The modulation of the fibre properties may take the form of a change in the refractive index of the core of the fibre, induced for example by exposure of the fibre to the output from a UV laser, a femtosecond pulsed laser or a $\mathrm{CO} 2$ laser, or by a perturbation to the diameter of the fibre. The efficiency of coupling, and thus depth of the resonance bands, is determined by the "strength" of the grating (e.g the amplitude of the refractive index modulation), the overlap of the mode profiles of the modes of the core and cladding, and the length of the grating. Environmental parameters that can influence the period of the gratings and/or the difference between the effective refractive indices of the core and cladding modes can be sensed by monitoring the concomitant changes in the central wavelength of the resonance bands.

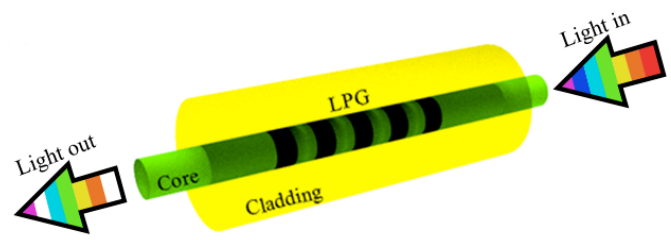

(a)

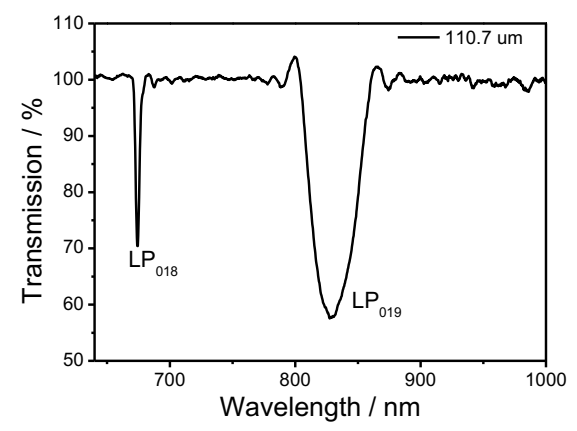

(b)

(c)

Figure 1: (a), schematic illustration of the LPG optical fibre, shown the attenuation bands introduced to the broad input spectrum; (b), transmission spectrum, measured in air, of an LPG period of $110.7 \mu \mathrm{m}$ and length $30 \mathrm{~mm}$ fabricated in an optical fibre of cut off wavelength $670 \mathrm{~nm}$, coupling to the $\mathrm{LP}_{018}$ and $\mathrm{LP}_{019}$ cladding modes. Adopted from [1].

\section{LPGs as a chemical sensing platform.}

Among the wide variety of optical fibre based sensor designs and measurement schemes that have been reported and demonstrated for chemical sensing, refractometers and chemical sensors based on optical fibre gratings have attracted considerable interest, in part because they offer wavelength-encoded information, which overcomes the referencing issues associated with intensity based approaches. Such devices have been investigated extensively $[2,3,4]$.

The sensitivity of LPGs to the refractive index of their surrounding medium arises from the dependence of the phase matching condition (equation 1) upon the effective refractive index of the cladding mode. This sensitivity may be used to measure the refractive index of a solution, providing information on its concentration, but this is not chemically selective. To provide the sensor with selective response to specific analytes, the cladding of the region of the optical fibre containing the LPG can be coated with a material that exhibits a change in its optical thickness in response to a target analyte. Similarly to surface plasmon resonance and 
planar waveguide devices, LPG have been shown to provide highly precise analytical information about adsorption and desorption processes associated with the RI and thickness of the sensing layer.

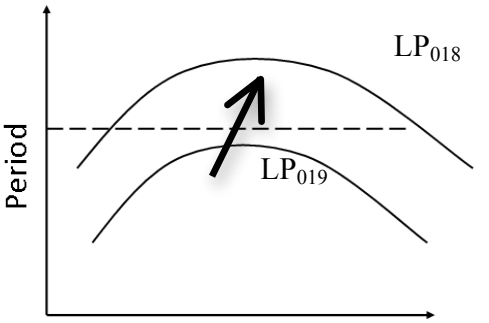

Wavelength

(a)

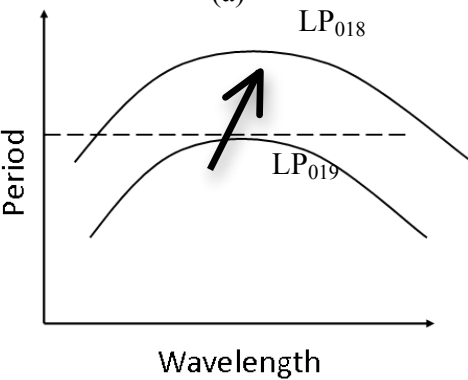

(c)

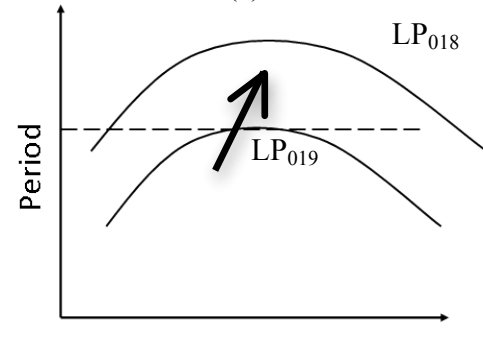

Wavelength

(e)

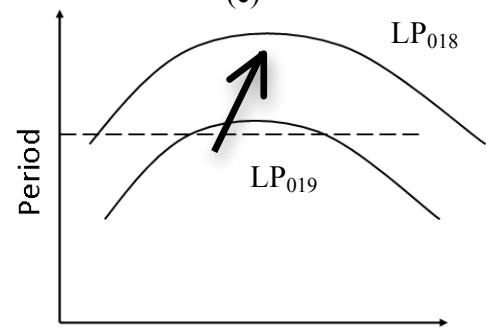

Wavelength

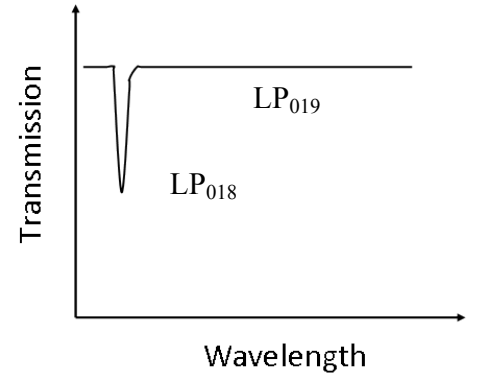

(b)

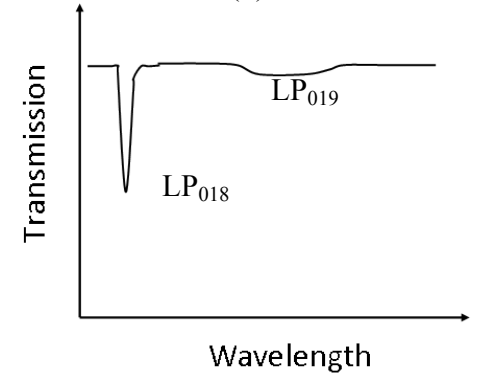

(d)

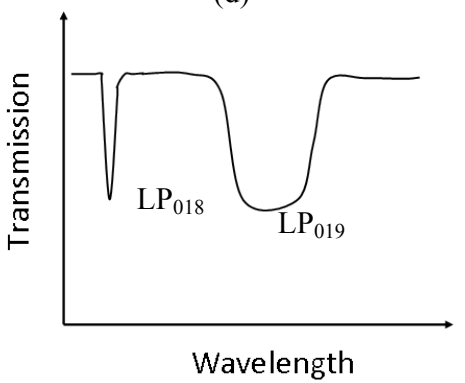

(f)

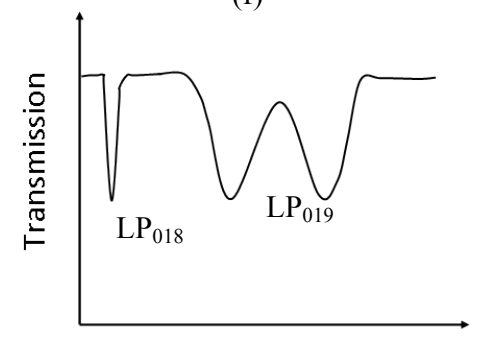

Wavelength

Figure 2: Illustration of the properties of an LPG near the phase matching turning point (PMTP); arrow shows direction of the shift of the phase matching curve at the increase of the effective refractive index of the cladding mode caused by increasing optical thickness. Adapted from [5]. 
The phase matching condition in equation 1 can be used to generate a family of curves that allow the prediction of the resonance wavelengths by calculating the dispersion of the core and cladding modes. The phase matching curves can be used to explain the way in which LPGs can be used as a chemical sensing platform.

For higher order cladding modes the curves contain a turning point, the so-called phase matching turning point, PMTP, or turn around point, TAP. Due to the gradient of the curves, the resonances bands of LPGs fabricated to access the phase matching turning point exhibit their maximum sensitivity to external perturbations [6]. The evolution of the transmission spectrum of an LPG at the PMPT in response to, for example, changes in the optical thickness of a coating despotised on the optical fibre can be understood with reference to figure 2 . The LPG is fabricated with a period such that, without a coating, it is not possible to couple to the $\mathrm{LP}_{019}$ mode. The corresponding spectrum contains a resonance band for coupling to the $\mathrm{LP}_{018}$ band (figures 2(a) and (b)). As the optical thickness (product of the geometrical thickness and refractive index) of the coating increases, the effective index of the cladding mode is changed, causing the phase matching curves move in the direction of the arrow shown in figure 2(a). When the horizontal line corresponding to the LPG period is tangential to the phase matching curve at the PMTP, a broad resonance band corresponding to coupling to $\mathrm{LP}_{019}$ begins to form, and there is a small blue shift in the central wavelength of the $\mathrm{LP}_{018}$ resonance band. Further increases in the optical thickness of the coating result in conditions at which the coupling to the same cladding mode occurs at 2 different wavelengths as the further development of the $\mathrm{LP}_{019}$ resonance band causes it to split into two bands, the so called dual resonance [6]. LPGs of appropriate period, coated with functional materials and operating at the PMTP, have been used to demonstrate sensors for chemical analytes in gaseous [7] or liquid media [5].

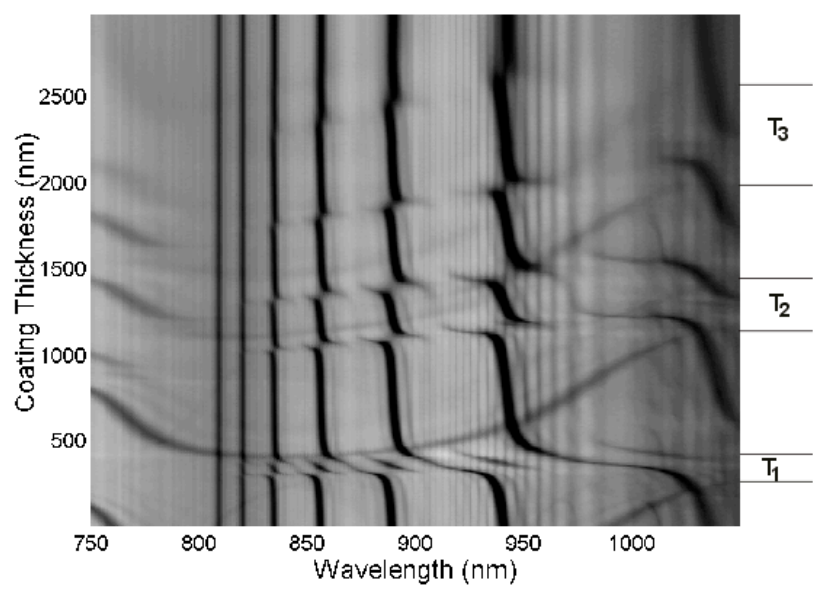

Figure 3: Evolution of the spectrum of an LPG of period 400 microns fabricated in borongermanium co-doped fibre in response to the deposition of a coating of $\omega$-tricosanoic acid (refractive index 1.57). Black represents $0 \%$ transmission, and white $100 \%$ transmission. The labels T1, T2 and T3 refer to mode transition regions. All spectra recorded with the LPG in air. Adapted from [8].

The deposition of a thin coating onto an LPG causes a further phenomenon that has a significant influence on the sensitivity of the resonance bands to changes in the properties of the coating. The evolution of the transmission spectrum of an LPG of period $400 \mu \mathrm{m}$ (not at the PMTP) to the deposition of such a coating is shown in figure 3 [8]. Here, the responses of 
the resonance bands to an increase of the coating thickness are characterised by blue wavelength shifts, which accelerate as the coating thickness increases (region $\mathrm{T}_{1}$ ). The region of high sensitivity to coating thickness is termed the "mode transition region". As the thickness is increased further, movement of the bands slows, with the band taking on the characteristics of the adjacent lower order resonance band. Further mode transition regions are observed with increasing coating thickness.

The physical processes underlying this behavior were clarified by a number of theoretical treatments $[9,10,11,12,13,14]$. Using an LP mode approximation, it has been shown that, as the optical thickness of the coating is increased, the coating becomes phase matched with one of the cladding modes. This is accompanied by a rapid change in the effective index of the mode, as shown in figure 4 [12]. The loss of the cladding mode results in a reorganization of the remaining cladding modes, such that the effective index, and the electric field profile, of each changes to become that of the adjacent lower order mode [14].

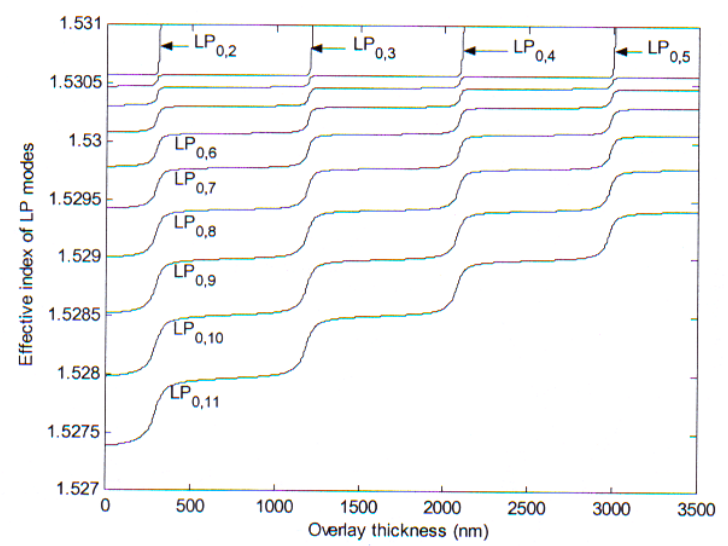

Figure 4: Effective refractive indices of the cladding modes, calculated using the LP approximation, as a function of overlay thickness, illustrating the reorganisation of the cladding modes during the transition to guidance by the overlay. Adapted from [12].

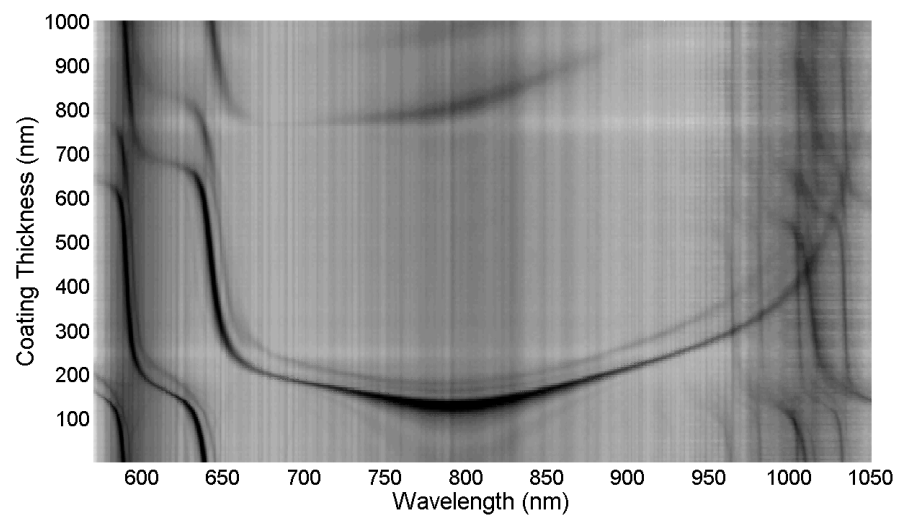

Figure 5: Evolution of the transmission spectrum of an LPG of period $100 \mu \mathrm{m}$, fabricated in a single mode optical fibre of cut-off wavelength $700 \mathrm{~nm}$, in response to the deposition of a coating of $\omega$ - tricosanoic acid using the Langmuir Blodgett Technique. Spectra recorded with the LPG below the water sub-phase. Adapted from [6]. 
By careful selection of the period of the LPG it is possible to ensure that, for a particular coating material, the PMTP and the mode transition region can coincide, offering optimized sensitivity to changes in the optical thickness of the coating, as illustrated in figure 5 [6].

\section{FABRICATION OF LPGs}

The modulation of the optical properties of the fibre required to fabricate an LPG may be achieved by modifying periodically the refractive index of the core by photoinduction or by physically deforming the optical fibre. The refractive index of the core may be modified by exposing locally the fibre to the output from a high-powered UV laser [15,16], a $\mathrm{CO}_{2}$ laser $[17,18]$, an infrared femtosecond laser [19], or by ion implantation [20]. Deformation of the fibre can be caused mechanically (e.g., inducing physical periodic corrugations on the fibre) [21] or by periodically tapering the fibre using a $\mathrm{CO} 2$ laser [22] or an electrical arc discharge [23] as the heat source. In recent years there have been considerable advances in the fabrication of LPGs using electric arc [24] and CO2 lasers[25], as they offer the ability to fabricate gratings in fibres that are not photosensitive - for example photonic crystal fibres and fibres with pure silica cores. The also allow the fabrication of short LPGs, of length as low as 2 periods. In the work described in this chapter, the LPGs have been fabricated via UV laser based inscription, as is allows gratings with shorter periods to be fabricated, allowing coupling to higher order cladding modes and thus access to the PMTP.

The inscription of an LPG via UV irradiation can be carried out via a point-by-point method [26] or by using an amplitude mask [26], as illustrated in figure 6. An amplitude mask introduces a spatial modulation to the intensity of the laser beam incident on the optical fibre, figure 6(a). In the point-by-point technique (figure 6(b)), the LPG is formed by exposing a section of length equal to half the period of the LPG to the UV beam for a set time and then translating either the fibre or the laser beam by a distance equal to the period, repeating the process until the desired length is attained. Although the fabrication of LPGs using the pointby-point approach can be more time consuming than using an amplitude mask, the flexibility of the technique allows the user to create LPGs with arbitrary period, with non-uniform period, and with arbitrary refractive index modulation profile by varying the dwell time at each point.

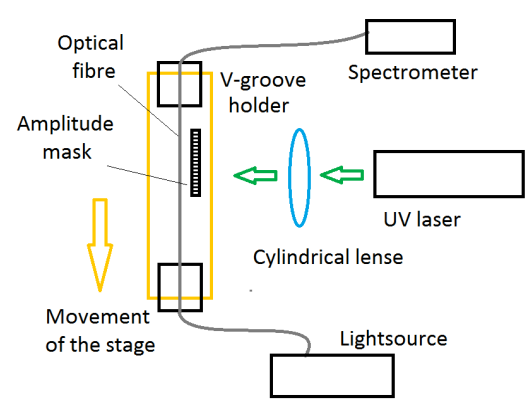

(a)

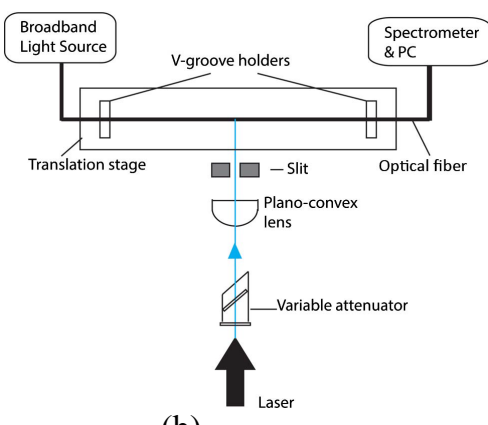

(b)

Figure 6: Schematic illustration of the LPG fabrication using UV laser beam via (a), amplitude mask; and (b), point-by-point technique. Adapted from [27].

It is known that optical fibre LPGs exhibit their highest sensitivity to environmental perturbation when the period is such that the phase matching condition is satisfied at its turning point. The reproducible fabrication of LPGs with parameters satisfying this condition requires high resolution control over the properties of the grating. The performance of an LPG fabrication system based on the point-by-point UV exposure approach was analysed by Wong 
et al [27]. It was demonstrated that the control of factors influencing reproducibility, such as period, duty cycle, and the environment in which the device is fabricated, is extremely important. In particular, the changes in grating period as small as $0.1 \mu \mathrm{m}$ have a significant influence on the final transmission spectrum of the LPG sensor as shown in figure 7(a).

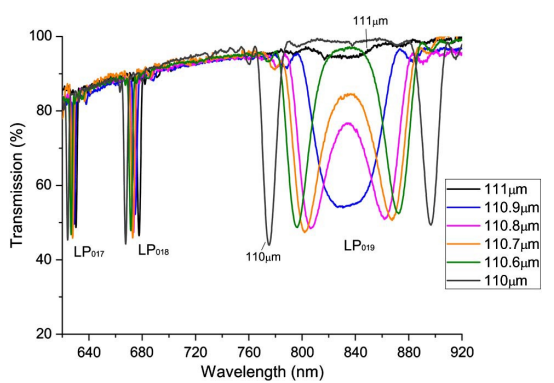

(a)

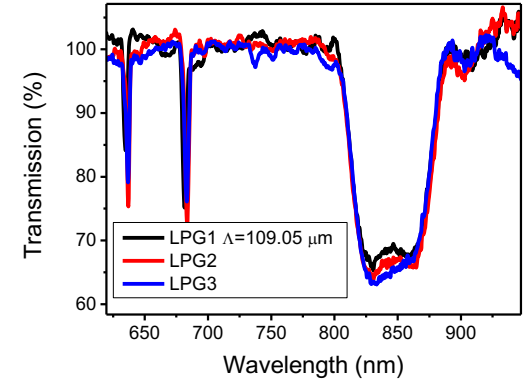

(b)

Figure 7: Transmission spectra of LPGs at and around the PMTP with different grating periods written using (a), point-by-point technique [27]; (b) Transmission spectra of LPGs at the PMTP with the same grating period written using amplitude mask.

The repeatability of the fabrication procedure is one of the important requirements for mass production of LPG based sensors and it is highly challenging to achieve this especially when LPG operates close to or at the PMTP. The repeatability of the point-by-point LPG fabrication system was investigated by fabricating four LPGs of nominally identical period in [27]. Once the period of the LPG at which the optimum sensitivity for the particular application has been identified, amplitude masks can be used for reliable and reproducible mass production. It should be noted that optimum sensitivity of the LPG for the particular application depends on the bulk RI range at which sensor needs to operate, on the coating used to sensitize the LPG and the range of the analyte that needs to be measured. Figure $7 \mathrm{~b}$ shows the repeatability achieved using an amplitude mask. The main advantage of the amplitude mask over point-bypoint approach is the reduced fabrication time. For same parameters of the writing laser, length and the strength of the LPG, the average fabrication time can be reduced from hours to minutes when compare amplitude mask with point-by-point approach [28].

\section{SURFACE MODIFICATION}

The combination of optical fibres and nanomaterials provides the prospect for the fabrication of chemical sensors with high sensitivity and that offer specific response to targeted chemical species $[29,30]$. Achieving the coating thickness that provides optimized sensitivity requires control on the $\mathrm{nm}$ scale, which is why many reports have exploited the Langmuir Blodgett (LB) and layer-by-layer (LbL) electrostatic self-assembly (ESA) coating deposition techniques, where a multilayer coating is deposited with each layer having a thickness of order $1 \mathrm{~nm}$. Based on this principle, sensors for organic vapors, metal ions, humidity, organic solvents and biological materials have been reported [31, 32, 33].

\subsection{Layer by-layer electrostatic self-assembly of mesoporous thin films}

The $\mathrm{LbL}$ technique, which is based on the alternate adsorption of polycations and polyanions onto the surface of a substrate, is a powerful surface modification method, figure 8 . This alternate adsorption technique is still expanding its potential because of its versatility and 
convenience for the fabrication of nano-assembled thin films employing various organic and inorganic materials.

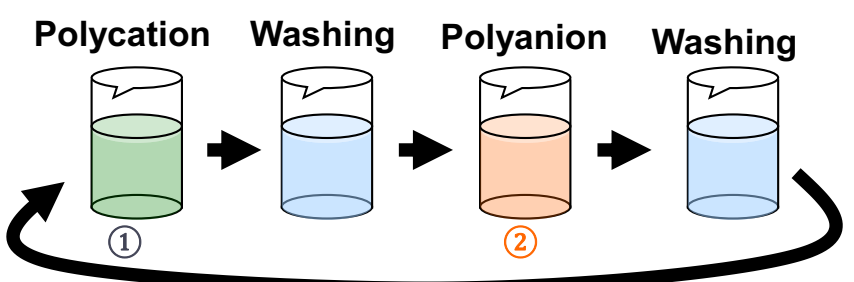

Figure 8: Schematic illustration of the layer-by-layer electrostatic self-assembly method.

We have demonstrated an approach to LPG based chemical sensing that consists of a 2 stage process. The first stage involves the deposition of a mesoporous coating onto an LPG operating near the phase matching turning point. In the second stage, a functional material, chosen to be sensitive to the analyte of interest, is infused into the base mesoporous coating. The mesoporous thin film be used for sensing utilising functional groups of the polycation [34] or a functional compound can be infused into the film, increasing the range of possible applications [5]. The initially low RI of the mesoporous coating, 1.2@633 nm, can be increased significantly by the chemical infusion, resulting in a large change in the LPG's transmission spectrum.

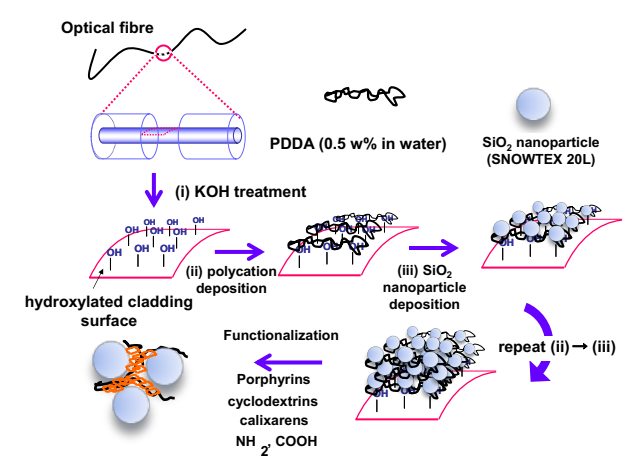

Figure 9: Schematic illustration of the deposition of a mesoporous coating onto the LPG fibre. Adapted from [5].

In a typical procedure, $\mathrm{SiO}_{2} \mathrm{NPs}$ are deposited onto the surface of the fibre using the LbL process, as illustrated in figure 9 [5]. Briefly, the section of the optical fibre containing the LPG, with its surface treated such that it is terminated with $\mathrm{OH}$ groups, is alternately immersed into a $0.5 \mathrm{wt} \%$ solution containing a positively charged polymer, Poly(diallyldimethylammonium chloride), PDDA, and, after washing, into a $1 \mathrm{wt} \%$ solution containing the negatively charged $\mathrm{SiO}_{2}$ NPs solution, each for $20 \mathrm{~min}$. This process is repeated until the required coating thickness is achieved. The coated fibre is immersed in a solution of, for example, Tetrakis(4-sulfophenyl)porphine, TSPP, or polyacrylic acid, PAA, as functional compound for $2 \mathrm{~h}$, which infuses into the porous coating and provides the sensor with its specificity. Due to the electronegative sulfonic groups present in the TSPP and carboxylic in PAA, an electrostatic interaction occurs between the infused compound and the positively charged PDDA in the (PDDA/ $\left.\mathrm{SiO}_{2}\right)_{\mathrm{x}}$ film (where $\mathrm{x}$ subscript denotes number of bilayers) [5]. PAA is considered as a promising sensor element for ammonia sensing, owing to the presence of free carboxylic function groups that lead to high affinity towards amine compounds [35]. On the other hand it has been shown that protonation and deprotonation of TSPP leads to the 
changes in its optical properties [5]. After immersion into the TSPP or PAA solutions, the fibre is rinsed in distilled water, in order to remove physically adsorbed compounds, and is dried by flushing with $\mathrm{N}_{2}$ gas. The compounds remaining in the porous silica structure are bound to the surface of the polymer layer that coats each nanosphere. This effectively increases the available surface area for the compounds to bond to. The presence of functional chemical compounds increases the RI of the porous coating and results in a significant change in the LPG's transmission spectrum [34], consistent with previous observations for increasing the coating thickness [6].

\subsection{Molecular imprinting}

Molecular imprinting is one of the most promising approaches for achieving specific molecular recognition. The technique is versatile, as any compound with functional groups can, in principle, be imprinted in different porogens (either water or organic solvents). The sensing and transduction principles combined with the imprinting approach are used to make the imprinting process quantifiable, thus providing detailed information about the recognition phenomenon occurring on the imprinting interface. The basic concept of molecular imprinting is based on the creation of imprints of a template compound (i.e. a chemical molecule or biological species that needs to be detected - the analyte) in a polymeric matrix, achieved by its incorporation during the polymerization step. Molecular imprinting is achieved in three steps, the first being the complex formation of the template with selected functional monomers, followed by a polymerization step for creation of a matrix that fixes the monomers in the right position to interact with the analyte and with the final step being the removal of the template to leave behind cavities specific for the template, as illustrated in figure 10 [47].

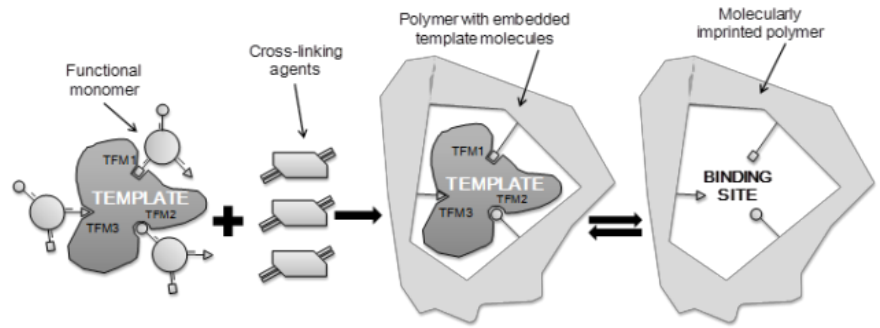

Figure 10: Schematic illustration of the molecular imprinting procedure. Adapted from [47].

A large body of work discussing the use of molecular imprinting can be found in the literature. Generally, the imprinting approach can be divided into two wide branches, based on the matrix material used for template incorporation. These approaches are discriminated by the use of organic and inorganic matrices.

\subsubsection{Organic MIPs}

To date, most examples of high fidelity molecular imprinting have used highly cross-linked organic polymers as the imprinting matrix [36]. Due to the variety of materials and the flexibility of the approach, molecular imprinting in organic matrices is more effective than it is in inorganic matrices [37]. Several approaches for the creation of MIP materials have been developed and are classified as covalent and non-covalent, depending on the nature of the binding between template and polymer matrix.

\subsubsection{Inorganic MIPs}

Molecular imprinting technique employing inorganic materials is generally based on the same principle as that of organic materials, consisting of covalent and/or non-covalent interaction between template and functional monomer, polymerization and template removal. Instead of 
organic monomer inorganic precursors, metal alkoxides, are usually used to form inorganic matrices via sol-gel process that incorporates template molecules, as shown in figure 11 [37].

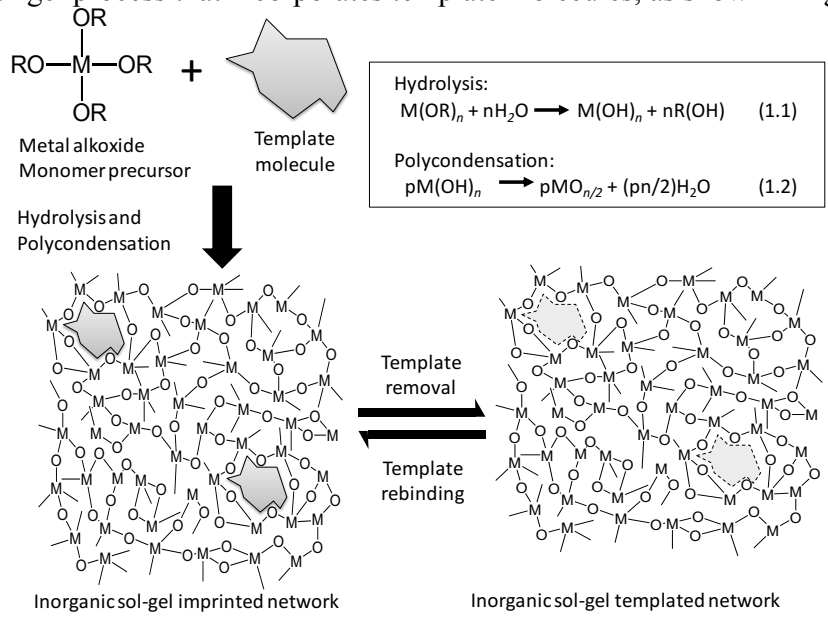

Figure 11: Schematic illustration of the molecular imprinting using sol-gel process; R-alkyl radical; $\mathrm{M}$ represents a network-forming element such as $\mathrm{Si}, \mathrm{Ti}, \mathrm{Zr}, \mathrm{Al}$ etc. Adapted from [37].

The ability to deposit such materials in the form of a thin film is of significance for sensor applications. In comparison with bulk materials, ultrathin films provide faster binding, better accessibility of the imprinted sites, higher binding efficiency and improved adsorption rate [37].

\section{EXPERIMENTAL RESULTS}

\subsection{Ammonia sensors}

An LPG of period $100 \mu \mathrm{m}$, with a mesoporous coating of PDDA/ $\mathrm{SiO}_{2}$ nanoparticles $(\mathrm{NPs}$ ) (SNOWTEX 20L (40-50 nm), Nissan Chemical) deposited using the LbL process and infused with TSPP as the functional compound, was used for the detection of ammonia in the gas phase and in solution [5].

For the detection of ammonia in solution, the LPG the deposition process used is illustrated in figure 12. The sensitivity to ammonia in water of an LPG coated with a TSPP infused $\left(\mathrm{PDDA} / \mathrm{SiO}_{2}\right)_{10}$ film was characterized by sequential immersion of the coated LPG into ammonia solutions with concentrations $0.1,1,5$ and $10 \mathrm{ppm}$. The lower ammonia concentrations were prepared by dilution of the stock solution of $28 \mathrm{wt} \%$. In order to assess the stability of the base line, the coated LPG was immersed several times into $150 \mu \mathrm{L}$ of pure water. The decrease of attenuation of the second resonance band, $\mathrm{LP}_{021}$, at $800 \mathrm{~nm}$, suggests the partial removal of the adsorbed TSPP molecules [5]. The equilibrium state was achieved after several exposures into water. For the ammonia detection, the LPG fibre was exposed into a $150 \mu \mathrm{L}$ ammonia solution of $0.1 \mathrm{ppm}$, followed by drying and immersion into ammonia solutions of 1,5 and $10 \mathrm{ppm}$ [5].

The response of the transmission spectrum to varying concentration of ammonia is shown in figure 12a. The dynamic response of the sensor was assessed by monitoring the transmission at the centre of the $\mathrm{LP}_{021}$ resonance band at $800 \mathrm{~nm}$. The response is shown in figure 12(b), where the "air", " $\mathrm{H}_{2} \mathrm{O}$ " and " $\mathrm{NH}_{3}$ " regions correspond to the transmission recorded at $800 \mathrm{~nm}$ after drying the LPG and immersing the device into water and ammonium solutions, respectively. After repeating the process of immersion in water and drying 4 times, the 
recorded spectrum was stable, demonstrating the robustness and stability of the employed molecules in aqueous environments $\left(\mathrm{H}_{2} \mathrm{O}\right.$ regions indicated in figure 12(b)). On immersion in $1 \mathrm{ppm}$ and $5 \mathrm{ppm}$ ammonia solutions, the transmission measured at $800 \mathrm{~nm}$ increased. The transmission when the coated LPG was immersed in a $10 \mathrm{ppm}$ ammonia solution exhibited a further increase, reaching a steady state within $100 \mathrm{~s}$, as shown in figure 12(b) [5]. The resonance feature corresponding to coupling to the $\mathrm{LP}_{020}$ cladding mode exhibited additional small red shifts of 0.5 and $1.5 \mathrm{~nm}$ when subsequently immersed in solutions of $1 \mathrm{ppm}$ and 10 ppm ammonia concentration, respectively, along with decreased amplitude, as shown in figure 9(a). The limits of detection (LOD) for the $100 \mu \mathrm{m}$ period LPG coated with a $\left(\mathrm{PDDA} / \mathrm{SiO}_{2}\right)_{10}$ film that was infused with TSPP were $0.14 \mathrm{ppm}$ and $2.5 \mathrm{ppm}$ when transmission and wavelength shift were measured respectively. The LOD was derived from the calibration curve and the following equation $[5,38]$.

$$
\mathrm{LOD}=3 \cdot \sigma / m
$$

where $\sigma$ is the standard uncertainty obtained as a symmetric rectangular probability [39]; $m$ is the slope of the calibration curve.

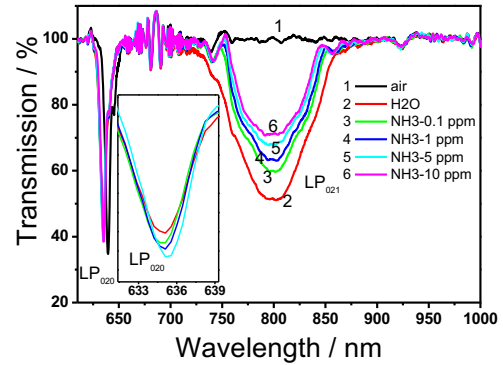

(a)

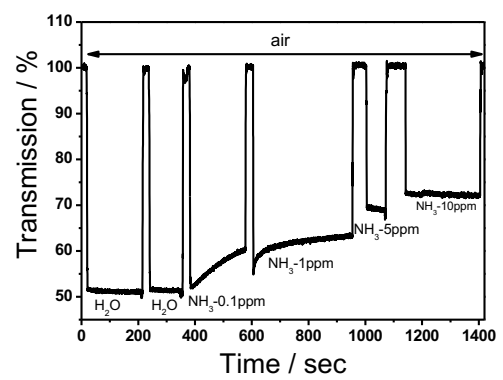

(b)

Figure 12: (a) Transmission spectra of the LPG coated with a TSPP -nfused (PDDA/SiO $\left.{ }_{2}\right)_{10}$ film due to immersion into water and into ammonia solutions of different concentrations: " $\mathrm{H}_{2} \mathrm{O}$ ", LPG exposed into water; "air", LPG in air after drying with $\mathrm{N}_{2}$ gas; " $\mathrm{NH}_{3} \mathrm{x} \mathrm{ppm",}$

LPG exposed into a $\mathrm{x}$ ppm ammonia solution, where $\mathrm{x}=0.1,1,5$ and 10. (b) Dynamic response to water and ammonia solutions $(0.1,1,5$ and $10 \mathrm{ppm})$ recorded at $800 \mathrm{~nm} ; \mathrm{LP}_{020}$ and $\mathrm{LP}_{021}$ are labelling the linear polarized 020 and 021 modes, respectively. Adapted from [5].

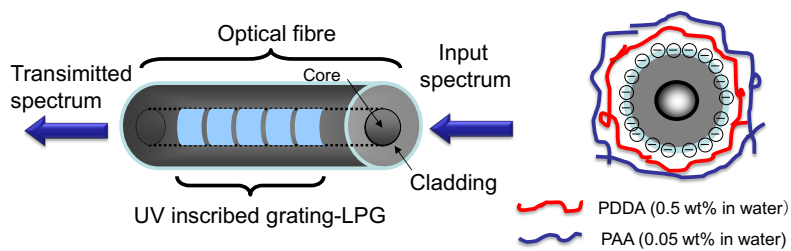

Figure 13: (a) Schematic illustration of an LPG and its surface modification using coating of PDDA and PAA deposited using the LbL approach. Adapted from [40].

For ammonia detection in gas phase, the LPG was coated, using the LbL approach with, an thin film composed of alternate layers of PAA, $\left(M_{\mathrm{w}}: 4000000\right)$ and PDDA, as shown in figure 13. PAA is a promising candidate for the creation of ammonia sensors, of which free carboxylic acid groups lead to the high sensitivity and selectivity toward amine compounds [40]. A quartz crystal microbalance (QCM) gas sensor based on the alternate deposition of $\mathrm{TiO} 2$ and PAA for the sensitive detection of amine odors has been reported, [35]. However, 
QCM sensors still have a weakness that the sensor response can be affected easily by humidity. The operation of the coated LPG sensor here is based on the acid-base interaction of amine odors to the $\mathrm{COOH}$ moiety of PAA under humid conditions, which showed no sensitivity to humidity.

On exposure of the coated LPG to increasing ammonia concentration, a linear wavelength shift of the resonance band was observed (figure 14(a)). The selectivity of the sensor was investigated using several amine and non-amine compounds (Figs. 14b). When the coated LPG was exposed to saturated non-amine compounds, no measurable changes were observed. The sensor demonstrated superior sensitivity towards ammonia as compared to other amine compounds (as saturated gases) such as triethylamine (TEA) and trimethylamine (TMA), which show relative wavelength shifts less than $40 \%$ of that measured in response to exposure to the ammonia gas at $100 \mathrm{ppm}$ [40]. The limit of section of the LPG sensor coated with the 7 layer thick film was $10.7 \mathrm{ppm}$ [7].

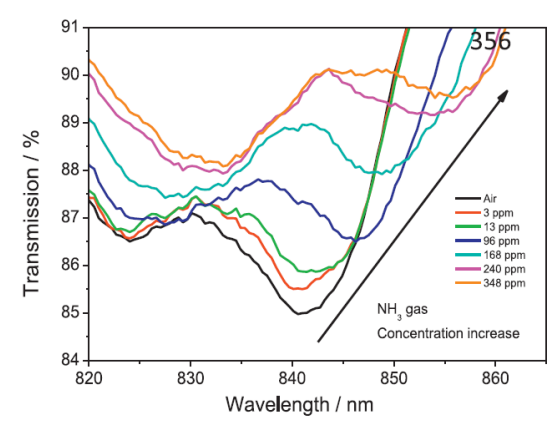

(a)

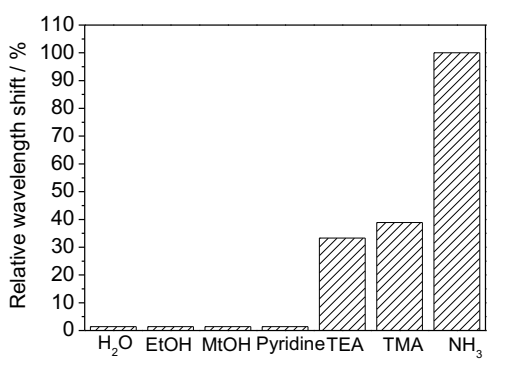

(b)

Figure 14: (a) Changes of the transmiossion spectrum of the LPG modified with a 7-cycle PAH/PAA film at the exposure to different concentrations of ammonia gas and (b) Relative wavelength shifts of the resonance bands in the TS of the LPG modified with a 7-cycle PAH/PAA film at the exposure to ammonia (100 ppm) and other analytes (saturated gas). Adapted from [7].

The detection of ammonia was also investigated usingan LPG of period $100 \mu \mathrm{m}$ that was coated with a multilayer film consisting of 15 layers of PDDA and TSPP (figure 15) [41]. This device exhibited a limit of detection of $0.67 \mathrm{ppm}$ for ammonia gas. TSPP molecules in the film acted as ammonia receptors because the TSPP morphologically changed from $\mathrm{J}$ - or $\mathrm{H}$ aggregates to free base monomers when it was deprotonated by being exposed to ammonia. It was found that $\mathrm{HCl}$ vapour could be used to increase the relative amount of $J$-aggregation in the TSPP and to restore the sensor response. The reversibility of the morphological change in the TSPP allowed reversible changes to occur in the properties of the coating, including the refractive index, film thickness and density, and electrostatic interactions; these influenced the transmission spectrum of the LPG [41]. 


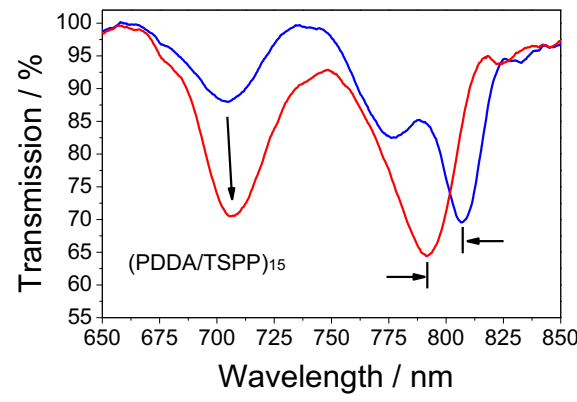

(a)

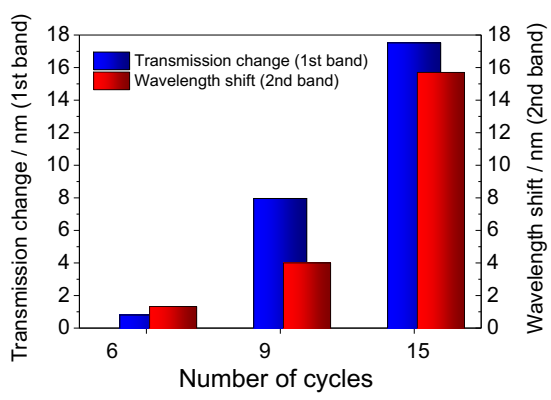

(b)

Figure 15: (a) Transmission spectra of LPG sensor of period $100 \mu \mathrm{m}$ and $4 \mathrm{~cm}$ length modified with PDDA/TSPP films produced using fifteen deposition cycles before (blue lines) and after (red lines) the sensors were exposed to $100 \mathrm{ppm}$ ammonia. (b) Comparison of the wavelength shifts and the transmission changes obtained using the sensors with films produced using six, nine, and fifteen deposition cycles. Adapted from [41].

\subsection{Detection of aromatic carboxylic acids}

The infusion of a functional compound into a mesoporous thin film coating an LPG can be used to tailor the selectivity of the LPG sensor. This section will provide several examples on how various functional compounds can be used to detect aromatic carboxylic acids [34], volatile organic compounds [44] and polyamines, which are cancer biomarkers. Aromatic carboxylic acids (ACAs) play an important role in the metabolizing process and their concentration in living organisms is strongly linked with some diseases [42]. For instance, a glycine conjugate of benzoic acid (BA) has been used in occupational medicine to monitor exposure to toluene [43]. The detection of ACAs using a coated LPG was reported in [34]. Mesoporous thin films were fabricated using a layer-by-layer self-assembly of poly(allylamine hydrochloride) (PAH) and $\mathrm{SiO}_{2}$ with a diameter in the range of $40-50 \mathrm{~nm}$ as described in section 5.1. The amino (NH2) functional group of PAH was used as binding site for the detection of ACAs. High sensitivity, with binding constants of $1.36 \pm 0.01 \times 10^{6}$ and $5.6 \pm 0.01 \times 10^{8} \mathrm{M}^{-1}$ for benzoic acid and mellitic acid, respectively, was achieved. The lowest measured concentration of $1 \mathrm{nM}$ of mellitic acid was achieved selectively over structurally (phenol) and functionally (acetic acid) related compounds.

The transmission spectrum of the $\left(\mathrm{PAH} / \mathrm{SiO}_{2}\right)_{10}$ film coated LPG was changed drastically by the adsorption of BA, as shown in figure 16(a). In particular, the resonance band at $663 \mathrm{~nm}$ corresponding to coupling to the $\mathrm{LP}_{020}$ cladding mode, shifted in wavelength by $0.5,1,1.5$ and $6 \mathrm{~nm}$ when exposed to BA with concentrations of $1,10,100$ and $1000 \mu \mathrm{M}$, respectively. In addition, a second resonance band corresponding to the coupling to the $\mathrm{LP}_{021}$ cladding mode developed at ca. $825 \mathrm{~nm}$, at the PMPT. As the concentration of BA increased, the change in the TS became more pronounced, indicating the increase of the amount of BA adsorbed inside the $\left(\mathrm{PAH} / \mathrm{SiO}_{2}\right) 10$ film. The sensor response was fast and saturated within $300 \mathrm{sec}$ for lower BA concentrations $(<1000 \mu \mathrm{M})$, as shown in figure 16(b). For the higher concentration $(1000$ $\mu \mathrm{M})$, however, the time to reach equilibrium was more than $1500 \mathrm{sec}$, indicating that interaction process was not completed for these values of concentration. 

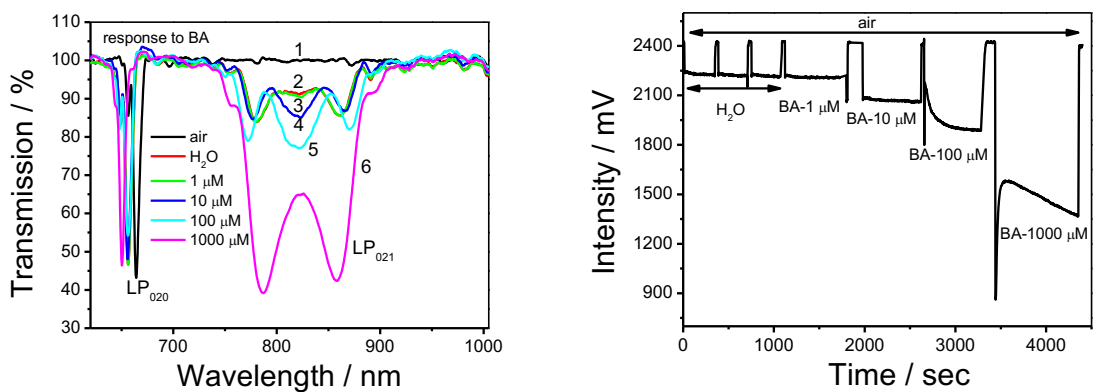

Figure 16: (a) Evolution of the transmission spectra of the optical fibre LPG coated with a $\left(\mathrm{PAH} / \mathrm{SiO}_{2}\right)_{10}$ film at different concentrations of BA and (b) time-dependence of the intensity measured at $825 \mathrm{~nm}$. Adapted from [34].

\subsection{Detection of polyamines}

The infusion of PAA into a mesoporous thin film consisting of of $\mathrm{SiO}_{2} \mathrm{NPs}$ and an organic moiety of a PAH polycation, was employed for the sensitive detection of a cancer biomarker, spermine (SPM), figure 17(a). The sensing mechanism is based on the measurement of the RI change induced by the binding of the SPM to the PAA. The lowest detected concentration of SPM was $1 \mu \mathrm{M}$ with a relatively fast, within few minutes, sensor response time achieved using an LPG modified with 5 cycles $\left(\mathrm{SiO}_{2} \mathrm{NPs} / \mathrm{PAH}\right)_{5} \mathrm{PAA}$ film, figure $17(\mathrm{~b})$.
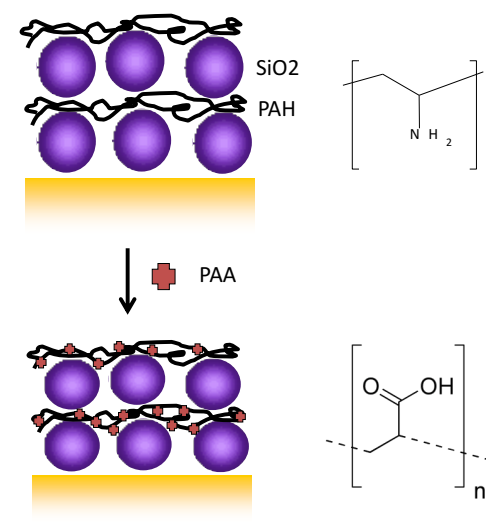

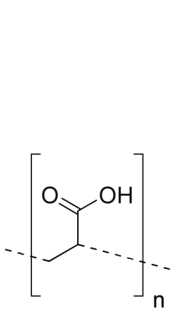

(a)

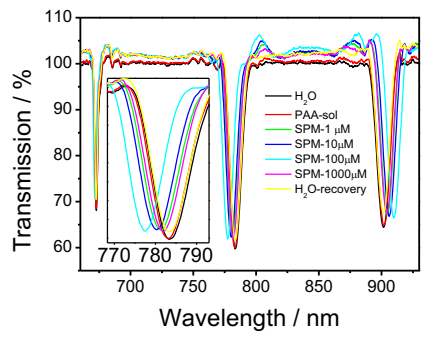

(b)

Figure 17: (a) Schematic illustration of the infusion of the PAA into $\mathrm{PAH} / \mathrm{SiO}_{2}$ thin film; and (b) evolution of the TS at the exposure to different concentrations of the SPM.

\subsection{Detection of VOCs}

An LPG modified with a mesoporous film of $\mathrm{SiO}_{2} \mathrm{NPs}$, infused with a calixarene as a functional compound, was employed for the detection of a volatile organic compounds (VOCs) mixture [44]. The sensing mechanism is based on the measurement of the RI change of the coating induced by the complexion of the VOCs with the calixarene. The LPG, modified with 5 cycles of $\mathrm{SiO}_{2} \mathrm{NPs} / \mathrm{PAH}$ that was infused with a calixarene[4], was exposed to chloroform, benzene, toluene and acetone vapours, figure 18. Calixarene molecules contain of a number of phenol or resorcinol aromatic rings connected together to a larger ring and the 
molecule is shaped like a bowl [45]. The analyte of the interest reacts with calixarene and becomes temporarily entrapped via gas state complexion. As only weak interactions occur (no covalent bond is created) the analyte is liberated easily from the cavity, with the result that the sensor is reversible. The sensitivity of the reaction depends on the morphology and charge of the molecule of interest and for this reason semi-specific reactions to different VOCs has been reported $[50,51]$.

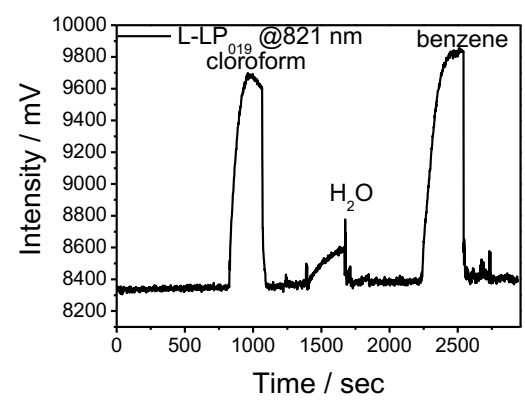

(a)

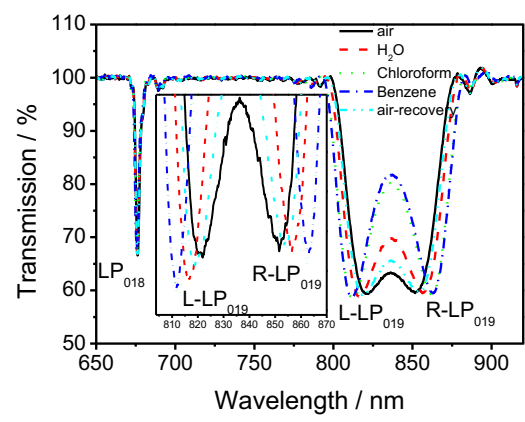

(b)

Figure 18: (a) Dynamic sensor response at the exposure to chloroform, water and benzene of the LPG with grating period of $111.5 \mu \mathrm{m}$ modified with the mesoporous $\left(\mathrm{PAH} / \mathrm{SiO}_{2}\right)_{5} \mathrm{C}[4] \mathrm{A}$

film measured $821 \mathrm{~nm}$ (b) TS spectra measured in atmospheres of: air, chloroform, water vapour and benzene. Adapted from [44].

\subsection{Development of biosensors}

The fast, reliable and highly sensitive detection of proteins and antigen-antibody reaction kinetics are desired in biology and medicine because it can facilitate prompt disease diagnosis. The presence of various proteins, or changes in their concentration, can be linked with alterations in physiology and act as an indicator of problems in the organism [46].

An LPG, modified with a coating of silica core gold shell $\left(\mathrm{SiO}_{2}: \mathrm{Au}\right)$ nanoparticles (NPs) (figure 19(a)) deposited using the layer-by-layer method, was employed for the development of a biosensor [1]. The $\mathrm{SiO}_{2}$ : Au NPs were electrostatically assembled onto the LPG with the aid of a PAH polycation layer. The LPG sensor was designed to operate at the phase matching turning point, to provide the highest sensitivity. The $\mathrm{SiO}_{2}$ : Au NPs were modified with biotin, which was used as a ligand for streptavidin (SV) detection. The sensing mechanism is based on the measurement of the refractive index change induced by the binding of the SV to the biotin. The effect on sensitivity of increasing the surface area by virtue of the $\mathrm{SiO}_{2}$ : $\mathrm{Au}$ nanoparticles' diameter and film thickness was studied. The lowest measured concentration of $\mathrm{SV}$ was $2.5 \mathrm{nM}$, achieved using an LPG modified with a 3 layer $\left(\mathrm{PAH} / \mathrm{SiO}_{2}: \mathrm{Au}\right)$ thin film composed of $\mathrm{SiO}_{2} \mathrm{NPs}$ of $300 \mathrm{~nm}$ diameter with a binding constant of $\mathrm{k}=1.7\left(\mathrm{pM}^{-1}\right)$, sensitivity of $6.9 \mathrm{~nm} /\left(\mathrm{ng} / \mathrm{mm}^{2}\right)$ and limit of detection of $19 \mathrm{pg} / \mathrm{mm}^{2}$, figure 19 (b). 

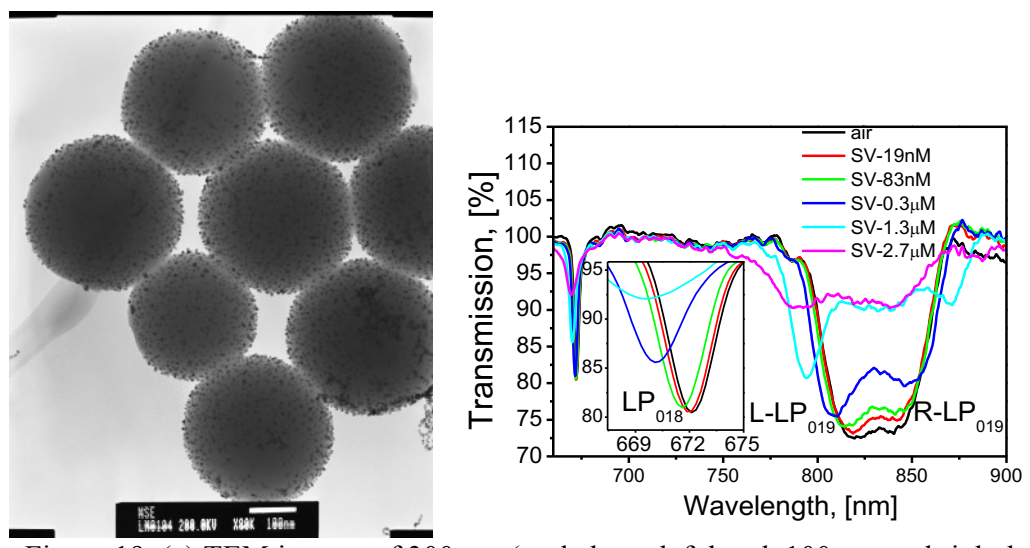

Figure 19. (a) TEM images of $300 \mathrm{~nm}$ (scale bars: left hand, $100 \mathrm{~nm}$ and right hand, $20 \mathrm{~nm}$ ); (b) Transmission spectrum of the $\left(\mathrm{PAH} / \mathrm{SiO}_{2(300 \mathrm{~nm})}: \mathrm{Au}\right)_{3}$ coated LPG modified on exposure to $\mathrm{SV}$ of different concentrations in: (a) air. Adapted from [1].

\subsubsection{Vancomycin detection}

An LPG modified with molecularly imprinted polymer nanoparticles (nanoMIPs) for the specific detection of antibiotics was reported in [47]. The operation of the sensor was based on the measurement of changes in refractive index induced by the interaction of nanoMIPs deposited onto the cladding of the LPG with free vancomycin (VA). The binding of nanoMIPs to vancomycin was characterised by a binding constant of $4.3 \pm 0.1 \times 10^{-8} \mathrm{M}$. The lowest concentration of analyte measured by the fibre sensor was $10 \mathrm{nM}$. In addition, the sensor exhibited selectivity, as much smaller responses were obtained for high concentrations $(\sim 700$ $\mu \mathrm{M})$ of other commonly prescribed antibiotics such as amoxicillin, bleomycin and gentamicin. In addition, the response of the sensor was characterised in a complex matrix, porcine plasma, spiked with $10 \mu \mathrm{M}$ of VA, figure 20 .

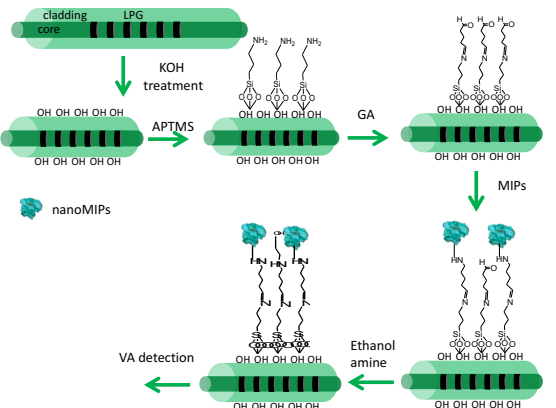

(a)

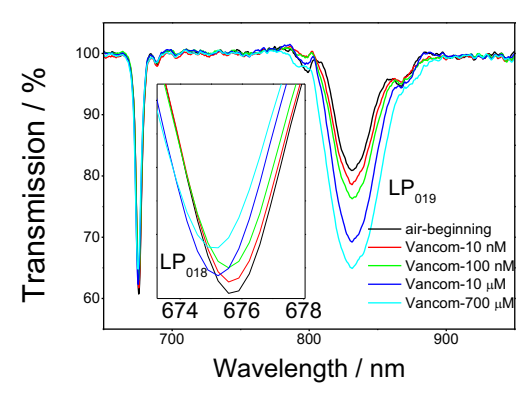

(b) 


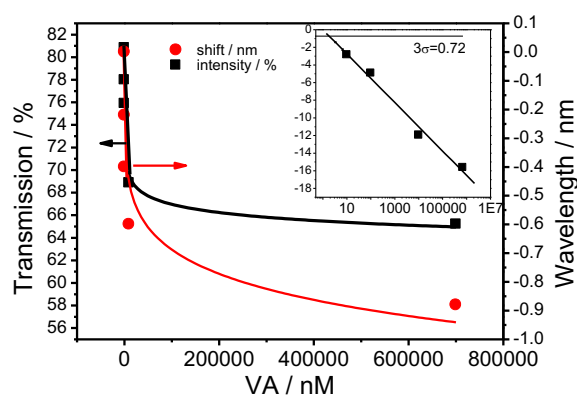

(c)

Figure 20: Schematic illustration of the protocol used to immobilize nanoMIPs. (a) Transmission spectrum of the nano-MIP coated LPG measured in air after exposure to VA concentrations ranging from $10 \mathrm{nM}$ to $700 \mu \mathrm{M}$; (b) Dependence of the transmission at the centre of the resonance band corresponding to coupling to the LP019 mode (square) and wavelength shift of the resonance band corresponding to coupling to the LP018 mode (circle) on the concentration of VA. The red and black lines are fits using the Langmuir adsorption isotherm. The inset shows the logarithmic dependence of transmission at the centre of the resonance band corresponding to coupling to the LP019 mode on VA concentration. Adapted from [47].

\subsection{Applications}

Examples of the practical application of the LPG sensors reported in literature are rather limited. This is related to the complexity in fabricating of the reliable and reproducible devices that can operate at optimal sensitivity regime, i.e., at the phase matching turning point. Perhaps one of the first examples of practical application of the LPG sensor was reported by Falate et al. [48] to measure the soybean oil concentration in samples obtained from the mixture of pure biodiesel and commercial soybean oil. The operation of the device was based purely on the sensitivity of the LPGs to the surrounding medium refractive index, which led to measurable modifications in the transmission spectrum. The sensor did not possess any specificity and was measuring only the RI change. The proposed analysis method results in errors in the oil concentration of $0.4 \%$ and $2.6 \%$ for pure biodiesel and commercial soybean oil, respectively. Techniques including total glycerol, dynamic viscosity, density, and hydrogen nuclear magnetic resonance spectroscopy were also employed to validate the method [48]. This section will describe the application of LPGs modified with sensitive thin films for measurement of indoor air quality (IAQ) and for assessment of the quality of beverages.

\subsubsection{Indoor air quality assessment using LPG sensors}

One of the key advantages of fibre optic sensors is the ability to multiplex an array of sensors, sensitive to the same or to different parameters. This can be of significant benefit in real environments, where the influence of interfering factors such as temperature or relative humidity should be reduced. Simultaneous detection of several parameters at the same location using a single optical fibre offers additional information that allows correction for changes of the interfering parameters [3].

Urrutia et al. [49] demonstrated simultaneous measurements of the humidity and temperature using novel dual-wavelength based sensing method. The LPG was partially coated with the humidity sensitive thin film in such a way that the main attenuation band was split into two 
different contributions allowing observing humidity and temperature change. The method however was based purely on the refractive index change.

Alternatively, a more tradition WDM approach can be adopted by fabricating an array of LPGs, each with different grating period, in a single optical fibre The LPGs can be functionalised to measure simultaneously several parameters, such as temperature, relative humidity and concentration of VOCs. The experimental arrangement is illustrated in figure 21 .

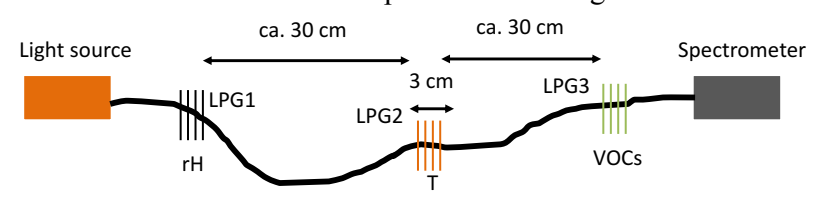

$\mathrm{LPG} 1=110.9 \mu \mathrm{m} \quad \mathrm{LPG} 2=110 \mu \mathrm{m} \quad \mathrm{LPG}=110.8 \mu \mathrm{m}$

Figure 21: Schematic illustration of the LPG sensor array. The individual LPGs were used to measure: LPG1 relative humidity (RH); LPG2 temperature; LPG3 volatile organic compounds (VOCs).

The LPGs have periods selected such that they all operate near the phase matching turning point and that differ by up to $1 \mu \mathrm{m}$ to facilitate wavelength division multiplexing, figure 22 . A mesoporous coating of silica nanospsheres was deposited onto LPG1, such that it was sensitive to RH. The surface of LPG2 was left unmodified and was used to measure temperature. A functional material, calixarene[4], was infused into a mesoporous silica nanospshere coating deposited onto LPG3 to sensitise the LPG to VOCs [50, 51].

The sensors were calibrated in the laboratory and the simultaneous measurement of the key indoor air quality parameters such as temperature, relative humidity and VOC was undertaken in laboratory and office environments. It was demonstrated successfully that the data produced by the LPG sensor array under real conditions was in a good agreement with that produced by commercially available sensors. The average differences between values obtained by the optical fibre sensor and standard temperature and $\mathrm{RH}$ sensors were better than $0.5^{\circ} \mathrm{C}$ and $5 \%$ respectively, figures 23 . Further, the potential application of fibre optic sensors for VOCs detection at high levels has been demonstrated.

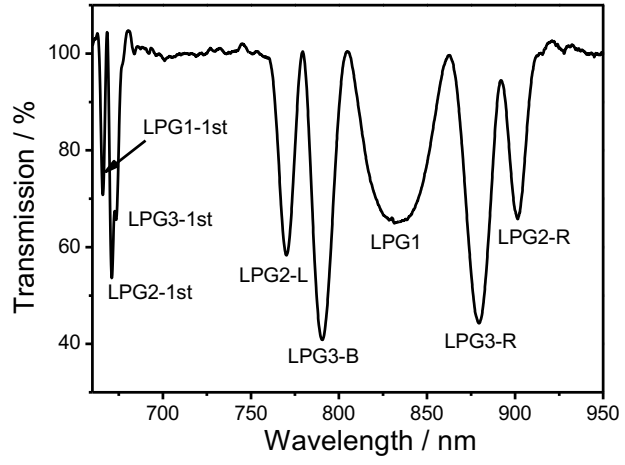

Figure 22: Transmission spectrum of the optical fibre LPG sensor array containing 3 LPGs of period 110.9 (LPG1), 110.0 (LPG2) and $110.8 \mu \mathrm{m}$ (LPG3) (L and R labels indicate short and long wavelength, respectively, positions of the resonance bands corresponding to the coupling of the same cladding mode, $1^{\text {st }}$ indicates the positions of first resonance bands). 


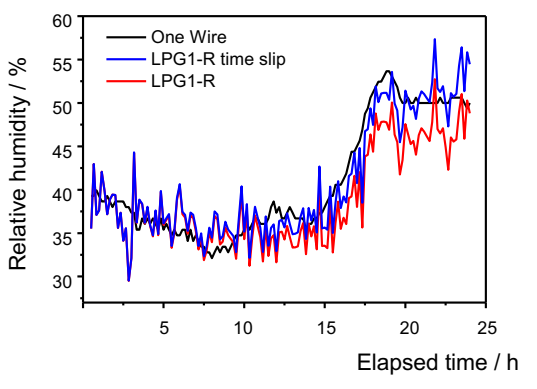

(a)

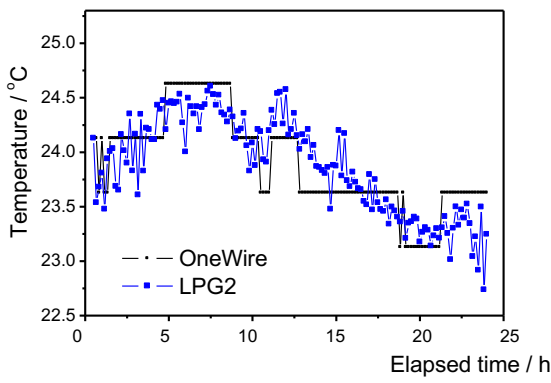

(b)

Fig 23: (a) Change of the RH measured using LPG sensor array; RH values were calculated from wavelength shift using calibration curves (use LPG1-R, figure 7): black line, RH measured using humidity and temperature data logger; blue line, with and red line, without use of time slip correction; and (b), blue line, change of the temperature measured using LPG sensor array; temperature values were calculated from wavelength shift using calibration curves (use LPG2-R); black line, temperature measured using temperature data logger.

\subsubsection{Beverage quality assessment}

An LPG functionalized with a mesoporous thin film was employed for the identification and quality assessment of beverages [52]. The principle of the discrimination of beverages using an LPG sensor is based on the measurement of the change in refractive index of a sensitive film, induced by the binding of the chemical compounds present in the beverage. The sensitive film deposited onto the LPG consisted of $\mathrm{PAH}$ and $\mathrm{SiO}_{2}$ NPs with diameters ranging from 40 $\mathrm{nm}$ to $50 \mathrm{~nm}$. PAH imparts selectivity, while the $\mathrm{SiO}_{2} \mathrm{NPs}$ endow the film with high porosity and enhanced sensitivity. In this study, five different types of beverages, red and white wines, brandy, nihonshyu (sake, a Japanese rice wine), and shochu (a Japanese distilled beverage), prepared via distillation and fermentation, were used to assess the capability of the sensor to identify the origin of the beverages. In addition, a selection of red wines was used to evaluate the use of the sensor in the assessment of the quality of beverages. The results obtained were benchmarked against those obtained using gas chromatography-mass spectrometry for the determination of volatile compounds contributing to the flavours of a set of red wines, figure 24(a). Principal component analysis (PCA) was employed for data analysis, figure 24(b). This approach enabled both quality assessment of beverages and identification of the methods and materials used for their preparation.
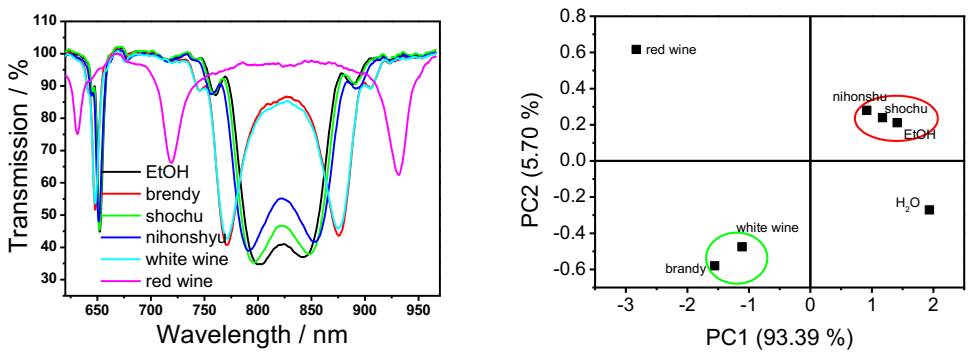

Figure 24: (a) Evolution of the transmission spectra of an the LPG coated with a $\left(\mathrm{PAH} / \mathrm{SiO}_{2}\right)_{10}$ film immersed in different beverages with the alcohol concentration adjusted to $10 \%$; black line, EtOH; red line, brandy; green line, shochu; blue line, nihonshu, cyan line, white wine; and magenta line, red wine. PCA performed using the data obtained with the LPG sensor. Adapted from [52]. 


\section{CONCLUSION}

In summary, in this chapter we have presented a comprehensive review of the development and fabrication of LPG chemical for application in sensing. The deposition of a sensitive coating onto the surface of an LPG creates a prospect for the development of highly sensitive sensor with the wide range of applications. LPG operating at the phase matching turning point has highest sensitivity to the range of measurands such as temperature, bending, strain and refractive index. Specificity, however, becomes an issue that limits practical application of the optical fibre LPG sensors operating at the PMTP. The possible solution for this is multiparameter measurement system that is based on the multiplexing of the array of sensors in the wavelength domain. This technique has been extensively employed in the interrogation of the fibre Bragg gratings. Its application with LPG hasn't been explored the same extent. The main reason for this is an ability to fabricate reliably and reproducibly LPGs at the PMTP. Fabrication methods based on the point-by-point and amplitude masks allow reliability and reproducibility of the LPGs, opening up opportunities for real world application of the LPG sensors. In this chapter we have provided examples of the practical application of LPG chemical sensors.

\section{REFERENCES}

[1]. Marques, L., Hernandez, F.U., James, S.W., Morgan, S.P., Clark, M., Tatam, R.P. Korposh S., "Highly sensitive optical fibre long period grating biosensor anchored with silica core gold shell nanoparticles", Biosens. Bioelectron. Vol. 75, 2015, pp. 222-231.

[2]. Grattan, K., Meggitt, B., "Chemical and environmental sensing", Kluwer Acad. Publisher, Boston.1999.

[3]. James, S.W., Tatam, R.P., "Optical fibre long-period grating sensors: characteristics and application", Meas. Sci. Technol. Vol. 14, 2003, pp. R49-R61.

[4]. James, S., Tatam R., "Fibre optic sensors with nano-structured coatings", Journal of Optics A: Pure and Applied Optics. 8, 2006, pp. S430-S444.

[5]. Korposh, S., Selyanchyn, R., Yasukochi, W., Lee, S.-W., James, S., Tatam, R., "Optical fibre long period grating with a nanoporous coating formed from silica nanoparticles for ammonia sensing in water", Materials Chemistry and Physics, Vol. 133, 2012, pp. 784-792.

[6]. Cheung, S.C., Topliss, S.M., James S.W., Tatam, R.P., "Response of fiber-optic longperiod gratings operating near the phase-matching turning point to the deposition of nanostructured coatings", Journal of the Optical Society of America B. Vol. 25, Issue 6, 2008, pp. 897-902.

[7]. Wang, T., Korposh, S., James, S., Tatam, R., Lee, S.-W., "Optical fibre long period grating sensor with a polyelectrolyte alternate thin film for gas sensing of amine odors", Sensors and Actuators B: Chemical. Vol., 185, 2013, pp. 117-124.

[8]. James, S. W., Cheung, C. S., Tatam, R. P., "Experimental observations on the response of 1st and 2nd order fibre optic long period grating coupling bands to the deposition of nanostructured coatings", Opt. Express, Vol. 15, 2007, pp. 13096-107.

[9]. Del Villar, I., Achaerandio, M., Matias, I.R., Arregui, F.J., "Deposition of overlays by electrostatic self-assembly in long-period fiber gratings", Opt.Lett. Vol. 30, 2005, pp. 720722.

[10]. Del Villar, I., Matias, I.R., Arregui, F.J., Achaerandio, M., "Nanodeposition of materials with complex refractive index in long-period fiber gratings", J. Lightwave Technol. Vol. 23, 2005, pp. 4192-4199.

[11]. Cusano, A., Ladicicco, A., Pilla, P., Contessa, L., Campopiano, S., Cutolo, A., Giordano, M., "Cladding mode reorganization in high-refractive-index-coated long-period gratings: effects on the refractive-index sensitivity", Opt. Lett. Vol. 30, 2005 pp. 25362538 . 
[12]. Del Villar, I., "Theoretical analysis and fabrication of nanostrctures with electrpstatic slef-assembly monolayer porcess" PhD Thesis, Universida Publica de Navarra, (2006). [13]. Wang, Z.Y., Helfin, J.R., Stoeln, R.H., Ramachandran, S., "Analysis of the optical response of long period fibre gratings to nm-thick thin-film coatings", Opt. Express, Vol. 13, 2005, pp. 2808-2813.

[14]. Del Villar, I., Matias, I.R., Arregui, F.J., "Influence on cladding mode distribution of overlay deposition on long-period fiber gratings", J. Opt. Soc. Am A., Vol. 23, 2006, pp. 651-658.

[15].Bhatia, V., Vengsarkar, A. M., "Optical fiber long-period grating sensors," Opt. Lett. Vol. 21, 1996, pp. 692-694.

[16].Blows, J., Tang, D. Y., "Gratings written with tripled output of Q-switched Nd:YAG laser," Electron. Lett. Vol. 36, 2000, pp. 1837-1839.

[17]. Davis, D. D., Gaylord, T. K., Glytsis, E. N., Kosinski, S. G., Mettler, S. C., Vengsarkar, A. M., "Long-period fiber grating fabrication with focused CO2 laser beams," Electron. Lett. Vol. 34, 1998, pp. 302-303.

[18]. Lan, X., Han, Q., Wei, T., Huang, J., Xiao, H., “Turn-aroundpoint long-period fiber gratings fabricated by CO2 laser pointby- point irradiations," IEEE Photon. Technol. Lett. Vol. 23, 2011, pp. 1664-1666.

[19].Kondo, Y., Nouchi, K., Mitsuyu, T., Watanabe, M., Kazansky, P., Hirao, K., "Fabrication of long-period fiber gratings by focused irradiation of infra-red femtosecond laser pulses," Opt. Lett. Vol. 24, 1999, pp. 646-648.

[20].Fujumaki, M., Ohki, Y., Brebner, J. L., Roorda, S., "Fabrication of long-period optical fiber gratings by use of ion implantation", Opt. Lett. Vol. 25, 2000, pp. 88-90.

[21].Savin, S., Digonnet, M. J. F., Kino, G. S., Shaw, H. J., "Tunable mechanically induced long-period fiber gratings", Opt. Lett. 25, 2000, pp. 710-712.

[22].Kakarantzas, G., Dimmick, T. E., Birks, T. A., Le Roux, R., Russell, P. S. J., "Miniature all-fiber devices based on $\mathrm{CO} 2$ laser microstructuring of tapered fibers", Opt. Lett. Vol. 26, 2001, pp. 1137-1139.

[23].Rego, G., Okhotnikov, O., Dianov, E., Sulimov, V., "High temperature stability of long-period fiber gratings using an electric arc", J. Lightwave Technol. Vol. 29, 2001, pp. $1137-1139$.

[24]. Rego, G. M., Marques, P. V. S., Santos, J. L., Salgado, H. M., "Arc-Induced longperiod gratings", Fiber Integrat. Opt. Vol. 24, 2005, pp. 245-59.

[25]. Wang, Y., "Review of long period gratings written by $\mathrm{CO} 2$ laser", Journal of Applied Physics. Vol. 108, 2010081101

[26]. Zhang, L., Zhang, W., Bennion, I., "In-fiber grating optic sensors," in Fiber Optic Sensors, S. Yin, P. B. Ruffin, and F. T. S. Yu, eds., 2nd ed. (CRC Press, 2008), pp. 109162.

[27]. Wong, R. Y. N., Chehura, E., Staines, S. E., James S. W., Tatam, R. P., "Fabrication of fiber optic long period gratings operating at the phase matching turning point using an ultraviolet laser", Applied Optics, Vol. 53, No. 21, 2014, pp. 4669-4674.

[28]. Hromadka, J., Correia, R., Korposh, S., "Fabrication of fiber optic long period gratings operating at the phase matching turning point using an ultraviolet laser via phase mask", Proc. SPIE, 2016, in press.

[29]. Del Villar, I., Achaerandio, M., Matias, I. R., Arregui, F. J. "Deposition of overlays by electrostatic self assembly in long-period fibre gratings", Opt. Lett. Vol. 30, 2005, pp. $720-722$.

[30]. Del Villar, I., Matias, I. R., Arregui, F. J., "Influence on cladding mode distribution of overlay deposition on long period fiber gratings", J. Opt. Soc. Am. A. Vol. 23, 2006, pp. 651-658. 
[31]. Cheung, S. C., Topliss, S. M., James, S. W,, Tatam, R. P., "Response of fibre optic long period gratings operating near the phase matching turning point to the deposition of nanostructured coatings", J. Opt. Soc. Am. B. Vol. 25, 2008, pp. 897-902.

[32]. Corres, J. M., Matias, I. R., del Villar, I., Arregui, F. J., "Design of pH sensors in long-period fiber gratings using polymeric nanocoatings", IEEE Sens. J. Vol. 7, 2007, pp. 455-463.

[33]. Keith, J., Hess, L. C., Spendel, W. U., Cox, J. A., Pacey, G. E., "The investigation of the behavior of a long period grating sensor with a copper sensitive coating fabricated by layer-by-layer electrostatic adsorption", Tatanta. Vol. 70, 2006, pp. 818-822.

[34]. Korposh, S., Wang, T., James, S., Tatam, R., Lee, S.-W., "Pronounced aromatic carboxylic acid detection using a layer-by-layer mesoporous coating on optical fibre long period grating", Sensors and Actuators B: Chemical, Vol. 173, 2012, pp. 300-309.

[35]. Lee, S.-W., Takahara, N., Korposh, S., Yang, D.-H., Toko, K., Kunitake, T., "Nanoassembled thin film gas sensors. III. Sensitive detection of amine odors using TiO2/poly(acrylic acid) ultrathin film quartz crystal microbalance sensors", Anal. Chem., Vol. 82, 2010, pp. 2228-2236

[36] Sellergren, B., Hall, A. J., "Molecularly Imprinted Polymers. Man-made Mimics of Antibodies and Their Applications in Analytical Chemistry" (ed. Sellegren, B.), Chapter 2 "Fundamental aspects on the synthesis and characterization of imprinted network polymers," Elsevier Science B.V., Amsterdam, 2003, pp. 21-57.

[37 ]. Lee, S.-W., Korposh, S., Selyanchyn, R., Kunitake, T., "Fundamentals and Perspectives of Molecular Imprinting in Sensor Applications", in: Handbook of Molecular Imprinting: Advanced Sensor Applications, Ed.: S.-W. Lee, T. Kunitake, Pan Stanford Publishing Pte Ltd, ISBN: 9789814316651, 2012.

[38]. Swartz, M.E., Krull, I.S., “Analytical Method Development and Validation”, Marcel Dekker, Inc.: NY USA; 1997.

[39]. Rafael, G., Possetti, C., Kamikawachi, R. C., Muller, M., Fabris, J. L., "Metrological evaluation of optical fiber grating-based sensors: An approach towards the standardization", J. Lightwave Technology (OFS-21). 2167500, 2011DOI 10.1109/JLT.2011.2167500

[40]. Wang, T., Korposh, S., Wong, R., James, S., Tatam, R., Lee, S.-W., "A novel ammonia gas sensing using a nanoassembled polyelectrolyte thin film on fiber optic long period gratings", Chemistry Letters, Vol. 41, No. 10, 2012, pp. 1297-1299.

[41]. Wang, T., Yasukochi, W., Korposh, S., James, S. W., Tatam, R. P., Lee, S.-W. “A long period grating optical fiber sensor with nano-assembled porphyrin layers for detecting ammonia gas", Sensors and Actuators B: Chemical, 2016, in press.

[42]. Sprague, C. L., Eelfarra, A. A., "Detection of carboxylic acids and inhibition of hippuric acid formation in rats treated with 3-butene-1,2-diol, a major metabolite of 1,3butadiene", Drug Metabolism and Disposition. Vol. 31, 2003, pp. 986-992.

[ 43 ]. Penner, N., Ramanathan, R., Zgoda-Pols, J., Chowdhury, S., "Quantitative determination of hippuric and benzoic acids in urine by LC-MS/MS using surrogate standards", J. Pharmaceut. Biomed. Anal. Vol. 52, 2010, pp. 534-543.

[44]. Korposh, S., Davis, F., James, S. W., Wang, T., Lee, S.-W., Higson, S., Tatam, R. P., "Detection of volatile organic compounds using an optical fibre long period grating with a calixarene anchored mesoporous thin film", Proc. SPIE 8794, 2013, pp. 87941I(4).

[45]. Hassan, A. K., Nabok, A. V., Ray, A. K., Lucke, A., Smith, K., Stirling, C. J. M., Davis, F., "Thin films of calix-4-resorcinarene deposited by spin coating and LangmuirBlodgett techniques: determination of film parameters by surface plasmon resonance," Mater. Sci. Eng., C, Vol. 8-9, 1999, pp. 251-255.

[46]. Wulfkuhle, J.D., Liotta, L. A., Petricoin, E. F., "Proteomic applications for the early detection of cancer", Nat. Rev. Cancer. Vol. 3, 2003, pp. 267-275. 
[47]. Korposh, S., Chianella, I., Guerreiro, A., Caygill, S., Piletsky, S. A., James, S. W., Tatam, R. P., "Selective vancomycin detection using optical fibre long period gratings functionalised with molecularly imprinted polymer nanoparticles", Analyst, Vol. 139, 2014, pp. 2229-2236.

[48]. Falate, R., Nike, K., Ramos da Costa Neto, P., Cação Jr. E., Muller, M., Kalinowski, H. J., Fabris, J. L., "Alternative technique for biodiesel quality control using an optical fiber long-period grating sensor", Química Nova, Vol. 30, No. 7, 2007, pp. 1677-1680.

[49]. Urrutia, A., Goicoechea, J., Ricchiuti, A. L., Barrera, D., Sales, S., Arregui, F. J., "Simultaneous measurement of humidity and temperature based on a partially coated optical fiber long period grating", Sens. and Act. B., Vol. 227, 2016, pp. 135-141.

[50]. Topliss, S.M., James, S.W., Davis, F., Higson, S.P.J., Tatam, R.P., "Optical fibre long period grating based selective vapour sensing of volatile organic compounds," Sens. Actuators, B, Vol. 143, No. 2, 2010, 629-634.

[51]. Korposh, S., Davis, F., James, S. W., Wang, T., Lee, S.-W., Higson, S., Tatam, R., P. "Detection of volatile organic compounds using an optical fibre long period grating with a calixarene anchored mesoporous thin film,” Proc. SPIE, 8794, pp. 87941I.

[52]. Korposh, S., Selyanchyn, R., James, S. W., Tatam, R. P., Lee, S.-W., "Identification and quality assessment of beverages using a long period grating fibre-optic sensor modified with a mesoporous thin film", Sensing and Bio-Sensing Research, Vol. 1, 2014, pp. 26-33. 


\title{
Long Period Grating Based Fibre Optic Chemical Sensors
}

\author{
Korposh, Sergiy
}

Springer

Korposh S, Lee SW, James S (2016) Long Period Grating Based Fibre Optic Chemical Sensors in Fiber Optic Sensors: Current Status and Future Possibilities (eds) Ignacio R. Matias, Satoshi Ikezawa, Jesus Corres, pp 241-267. Springer: Cham/Smart and in Sensors, Measurement and Instrumentation, Volume 21, 2017: DOI: 10.1007/978-3-319-42625-9_12

http://dx.doi.org/10.1007/978-3-319-42625-9_12

Downloaded from Cranfield Library Services E-Repository 